\title{
Porous Ferroconvection with Local Thermal Nonequilibrium Temperatures and with Cattaneo Effects in the Solid
}

\author{
I. S. Shivakumara ${ }^{a^{*}}$. M. Ravishab ${ }^{b}$ Chiu-On Ng ${ }^{c^{*}}$. V. L. Varun
}

\begin{abstract}
The influence of local thermal nonequilibrium (LTNE) with Cattaneo effects in the solid on the onset of thermal convective instability in a horizontal layer of Darcy porous medium saturated by a ferrofluid in the presence of a uniform vertical magnetic field is investigated. The presence of Cattaneo effect is to instil instability via oscillatory motion as well which is not reminiscent of the observed instability phenomenon in its absence. Increase in the value of solid thermal relaxation time parameter $\tau$ is found to advance the onset of oscillatory ferroconvection. The onset of stationary ferroconvection is delayed, while the onset of oscillatory convection is hastened with an increase in the value of inter-phase heat transfer coefficient $H_{t}$. The threshold value of $H_{t}$, at which the transition from stationary to oscillatory convection takes place, decreases with increasing $\tau$ noticeably and marginally with increasing magnetic parameter $M_{3}$, while it increases with increasing ratio of conductivities $\alpha$ and magnetic number $M_{1}$. The critical wave number for stationary convection is found to be higher than those of oscillatory convection.
\end{abstract}

Keywords: Cattaneo effects, Ferroconvection, Local thermal nonequilibrium, Porous medium

\section{${ }^{a}$ I. S. Shivakumara}

Department of Mathematics, Bangalore University, Bangalore 560 001, India

E-mail: shivakumarais@gmail.com

${ }^{\mathrm{b}}$ M. Ravisha

Department of Mathematics, Dr. G. Shankar Government Women's First Grade College and Post Graduate Study Centre, Ajjarakadu, Udupi-576101, India

E-mail: ravishmamatha@gmail.com

${ }^{*}$ Department of Mechanical Engineering, The University of Hong Kong, Pokfulam Road, Hong Kong, China

E-mail: cong@hku.hk 


\section{Introduction}

Ferrofluids are stable colloidal suspensions of magnetic nanoparticles in a nonmagnetic carrier fluid. The use of ferrofluids as a heat transfer medium stem from a possibility of controlling its flow and heat transfer process via an external magnetic field. Besides, magnetic forces are used to create circulation of coolant in small passages where natural convection is either absent or ineffective. Both theoretical and experimental developments of the subject are well documented in the literature (Rosensweig [1], Berkovsky et al. [2], Blums et al. [3], Hergt et al. [4], Alexiou et al.[5]). The magnetization of ferrofluids depends on the magnetic field, temperature and density. Hence, any variations of these quantities induce change of body force distribution in the fluid and eventually give rise to convection in ferrofluids in the presence of a gradient of magnetic field known as ferroconvection. Considering the prospect of heat transfer applications in electronics, micro and nanoelectromechanical systems and magnetically controlled heat transfer in energy conversion systems, ferroconvection in magnetized ferrofluids has triggered lot of research interest over the years (Odenbach, [6], Ganguly et al. [7], Kaloni and Lou [8]). Recently, Nkurikiyimfura et al. [9] reviewed the recent developments in this field. In particular, they have emphasized on thermal conductivity enhancement and thermomagnetic convection in devices using ferrofluids as heat transfer media.

Ferroconvection in a porous medium has also attracted considerable attention in the literature owing to its importance in controlled emplacement of liquids or treatment of chemicals, and emplacement of geophysically imageable liquids into particular zones for subsequent imaging etc. Besides, the subject of flow of ferrofluids through porous media is motivated by the potential use of ferrofluids to stabilize fingering in oil recovery processes. Rosensweig et al. [10] studied experimentally the penetration of ferrofluids in the Hele-Shaw cell. The stability of the magnetic fluid penetration through a porous medium in high uniform magnetic field oblique to the interface is considered by Zahn and Rosensweig [11]. The onset of ferroconvection in a porous medium in the presence of a vertical magnetic field is discussed by Vaidyanathan et al. [12] by employing the Brinkman equation while Qin and Chadam [13] carried out the nonlinear stability analysis of ferroconvection in a porous layer by including the inertial effects to accommodate high velocity. The laboratory-scale experimental results of the behaviour of ferrofluids in porous media consisting of sands and sediments have been presented by Borglin et al. [14]. Sunil and Amit Mahajan [15] used generalized energy method to study nonlinear 
convection in a magnetized ferrofluid saturated porous layer heated uniformly from below for the stress-free boundaries case. Shivakumara et al. ([16], [17]) investigated theoretically the onset of convection in a layer of ferrofluid saturated porous medium for various types of velocity and temperature boundary conditions. The onset of buoyancy-driven convection in a ferrofluid saturated sparsely packed porous medium with fixed heat flux condition at the lower rigid boundary and a general thermal boundary condition at the upper free boundary has been studied by Nanjundappa et al. [18].

In many practical applications involving hyper-porous materials and also media in which there is a large temperature difference between the fluid and solid phases, it has been realized that the assumption of local thermal equilibrium (LTE) is inadequate for proper understanding of the heat transfer problems. In such circumstances, the local thermal non-equilibrium (LTNE) effects are to be taken into consideration. Virto et al. [19] analyzed various causes of LTNE situations in detail. Under the circumstances, the recent trend in the study of thermal convective instability problems in porous media is to account for LTNE effects by considering a two-field model for energy equation each representing the fluid and solid phases separately. Realizing this fact, investigations have been carried out in the recent past to know LTNE effects on porous ferroconvection as well (Sunil et al. [20], Lee et al. [21], Shivakumara et al. ([22], [23]).

The energy equation used in the study of convective instability problems in a fluid/porous layer is a parabolic-type partial differential equation which allows an infinite-speed for heat transport. Nonetheless, the new theories make use of modified versions involve hyperbolic-type heat transport equation admitting finite-speed for heat transport. Thus, heat transport is viewed as a wave phenomenon rather than a diffusion phenomenon and this is referred to as second sound. In particular, the second sound effect appears greater in solids, especially those involved in porous metallic foams. A key way to introduce this effect is to use Cattaneo [24] law for the heat flux. Based on this approach, studies have been undertaken in the past to investigate thermal convection in an ordinary viscous fluid layer (Straughan and Franchi [25]; Lebon and Cloot [26]) and also in an ordinary viscous fluid-saturated porous medium using a local thermal equilibrium (LTE) model with Cattaneo-Fox and Cattaneo-Christov effects (Straughan [27]). The details about the developments on this topic are amply documented in the book by Straughan [28]. Recently, Straughan [29] investigated the effect of Cattaneo heat flux theory on thermal convection in a fluid-saturated Darcy porous medium using a local thermal 
non-equilibrium (LTNE) model for the first time. In addition to performing linear instability analysis, a global nonlinear stability threshold is determined and the effect of second sound is delineated in a detailed manner. Stranges et al. [30] examined natural convection of non-Fourier fluids of the single phase-lagging type which possess a relaxation time, reflecting the delay in the response of the heat flux and the temperature gradient. A review on thermal instability in a Brinkman porous medium incorporating fluid inertia and Cattaneo-Christov theory in the constitutive equation for heat flux is presented by Haddad [31] using LTE model. Recently, Shivakumara et al. [32] investigated the onset of thermal convection in a layer of fluid-saturated Brinkman porous medium taking into account fluid inertia and LTNE between the solid and fluid phases with Cattaneo effect in the solid.

However, no attention has been given to understand the influence of LTNE with Cattaneo effects in the solid on the onset of convection in a ferrofluid saturated porous layer heated from below. Since ferrofluids are used as heat carrier fluids in micro and nano devices, the proposed study is relevant in many heat transfer devices particularly to low- temperature fluids or fast heat transfer processes. Moreover, the Cattaneo effect on nanofluids has also been recognized in the literature and hence probing its influence on ferroconvection in porous media is considered to be relevant and important. The Cattaneo heat flux theory amounts to change the solid phase temperature equation from traditional parabolic to hyperbolic type which accounts for finitespeed heat transport in solids. As a result, thermal convective instability in a ferrofluid-saturated porous layer is found to occur via oscillatory mode under certain conditions. This is a novel result because instability is found to occur only via stationary convection in the absence of Cattaneo effect [21]. The results are presented graphically for a wide range of physical parameters and some of the convection systems previously reported in the literature is shown to be special cases of the present study.

\section{Mathematical Formulation}

The physical configuration is as shown in Fig. 1. We consider an initially quiescent incompressible constant viscosity ferrofluid saturated horizontal layer of Darcy porous medium of characteristic thickness $d$ in the presence of a uniform applied magnetic field $H_{0}$ in the vertical direction. The lower surface is held at constant temperature $T_{l}$, while the upper surface is at $T_{u}\left(<T_{l}\right)$. A Cartesian co-ordinate system $(x, y, z)$ is used with the origin at the bottom of the 
porous layer and the z-axis directed vertically upward in the presence of gravitational field. The solid and fluid phases of the porous medium are assumed to be in LTNE and a two-field model for temperatures is used. The solid temperature equation is modified to allow the heat transfer via a Cattaneo heat flux theory, while the usual Fourier heat-transfer law is used in the ferrofluid. The basic equations governing the flow of an incompressible ferrofluid saturating a layer of Darcy porous medium with LTNE and Cattaneo effects in the solid are (Straughan, [29], Nield and Bejan, [33]):

$$
\begin{aligned}
& \nabla \cdot \vec{q}=0 \\
& \vec{q}=-\frac{k}{\mu_{f}} \nabla \mathrm{p}+\frac{k \rho_{0}}{\mu_{f}}\left[1-\alpha_{t}\left(T_{f}-T_{a}\right)\right] \vec{g}+\frac{k \mu_{0}}{\mu_{f}}(\vec{M} \cdot \nabla) \vec{H} \\
& \varepsilon\left(\rho_{0} c\right)_{f} \frac{\partial T_{f}}{\partial t}+\left(\rho_{0} c\right)_{f}(\vec{q} \cdot \nabla) T_{f}=\varepsilon k_{f} \nabla^{2} T_{f}+h_{t}\left(T_{s}-T_{f}\right) \\
& (1-\varepsilon)\left(\rho_{0} c\right)_{s} \frac{\partial T_{s}}{\partial t}=-(1-\varepsilon)(\nabla \cdot \vec{Q})-h_{t}\left(T_{s}-T_{f}\right) \\
& \nabla \cdot \vec{B}=0 \\
& \nabla \times \vec{H}=0 \text { or } \vec{H}=\nabla \varphi
\end{aligned}
$$

where $\vec{q}=(u, v, w)$ the velocity vector, $p$ the pressure, $\vec{M}$ the magnetization, $\vec{H}$ the magnetic field intensity, $k$ the permeability of the porous medium, $\varepsilon$ the porosity of the porous medium, $\vec{B}$ the magnetic induction, $\vec{H}$ the magnetic field intensity, $\varphi$ the magnetic potential, $\mu_{f}$ the fluid viscosity, $\mu_{0}$ the magnetic permeability of vacuum, $T_{f}$ the temperature of the fluid, $T_{s}$ the temperature of the solid, $\vec{Q}$ the heat flux vector in the solid, $c$ the specific heat, $k_{f}$ the thermal conductivity of the fluid, $h_{t}$ is the inter-phase heat transfer coefficient, $\alpha_{t}$ is the thermal expansion coefficient, $T_{a}=\left(T_{l}+T_{u}\right) / 2$ is the average temperature, $\rho_{0}$ is the reference density and $\nabla^{2}=\partial^{2} / \partial x^{2}+\partial^{2} / \partial y^{2}+\partial^{2} / \partial z^{2}$ is the Laplacian operator. The term $\mu_{0}(\vec{M} \cdot \nabla) \vec{H}$ in Eq. (2) is the magnetic body force which appears as a result of polarization of the ferrofluid in the presence of magnetic field.

The Cattaneo's law for the solid heat flux is

$$
\tau_{s} \frac{\partial \vec{Q}}{\partial t}=-\vec{Q}-k_{s} \nabla T_{s}
$$

where $k_{s}$ is the thermal conductivity of the solid and $\tau_{s}$ is the solid thermal relaxation time. 
Further, $\vec{B}, \vec{M}$ and $\vec{H}$ are related by

$$
\vec{B}=\mu_{0}(\vec{M}+\vec{H}) \text {. }
$$

It is assumed that the magnetization is aligned with the magnetic field, but allowed a dependence on the magnitude of the magnetic field as well as temperature (Finlayson, [34]) and thus

$$
\vec{M}=M\left(H, T_{f}\right) \frac{\vec{H}}{H}
$$

where, $M=|\vec{M}|$ and $H=|\vec{H}|$. The magnetic equation of state, following (Finlayson, [34]), is taken as

$$
M=M_{0}+\chi\left(H-H_{0}\right)-K\left(T_{f}-T_{a}\right)
$$

where, $\chi=(\partial M / \partial H)_{H_{0}, T_{a}}$ is $\quad$ the magnetic $\quad$ susceptibility, $K=-\left(\partial M / \partial T_{f}\right)_{H_{0}{ }^{\prime} T_{a}} \quad$ is $\quad$ the pyromagnetic co-efficient and $M_{0}=M\left(H_{0}, T_{a}\right)$.

The basic state is quiescent and given by

$$
\begin{aligned}
\vec{q}_{b} & =(0,0,0), T_{f}=T_{f b}(z), T_{s}=T_{s b}(z), \vec{M}=\left(0,0, M_{b}(z)\right), \vec{B}=\left(0,0, B_{b}(z)\right), \\
\vec{H} & =\left(0,0, H_{b}(z)\right), \quad \vec{Q}=\left(0,0, Q_{b}(z)\right)
\end{aligned}
$$

where the subscript $b$ denotes the basic state. Substituting Eq. (11a) into Eqs. (2), (3), (4) and (7), we obtain

$$
\begin{aligned}
& \frac{d p_{b}(z)}{d z}=-\rho_{0}\left[1-\alpha_{t}\left(T_{f b}(z)-T_{a}\right)\right] g+\mu_{0} M_{b}(z) \frac{d H_{b}(z)}{d z} \\
& \nabla^{2} T_{f b}(z)=0 \\
& \nabla^{2} T_{s b}(z)=0 .
\end{aligned}
$$

Solving Eqs. (11c) and (11d) subject to the boundary conditions $T_{f b}=T_{s b}=T_{l}$ at $z=0$ and $T_{f b}=T_{s b}=T_{u}$ at $z=d$, we obtain

$$
T_{f b}(z)=T_{a}-\beta(z-d / 2)=T_{s b}(z)
$$

where, $\beta=\Delta T / d=\left(T_{l}-T_{u}\right) / d$ is the temperature gradient. Substituting Eq. (8) into Eq. (5) and using Eqs. (11a) and (11e), the basic state magnetic field intensity $\vec{H}_{b}(z)$ and magnetization $\vec{M}_{b}(z)$ are found to be (see Finlayson [34])

$$
\vec{H}_{b}(z)=\left[H_{0}-\frac{K \beta}{1+\chi}\left(z-\frac{d}{2}\right)\right] \hat{k}, \vec{M}_{b}(z)=\left[M_{0}+\frac{K \beta}{1+\chi}\left(z-\frac{d}{2}\right)\right] \hat{k}
$$


where $\hat{\mathrm{k}}$ is the unit vector in the vertical z-direction. Using Eqs. (11e) and (11f) in Eq. (11a) and integrating, we obtain

$$
p_{b}(z)=p_{0}-\rho_{0} g z-\frac{1}{2} \rho_{0} \alpha_{t} g \beta z(z-d)-\frac{\mu_{0} M_{0} K \beta}{1+\chi} z-\frac{\mu_{0} K^{2} \beta^{2}}{2(1+\chi)^{2}} z(z-d)
$$

where $p_{0}$ is the pressure at $z=0$.

\section{Linear Stability Theory}

To investigate the conditions under which the basic state is stable against small disturbances, we consider a perturbed state in the form

$$
\begin{aligned}
& \vec{q}=\vec{q}^{\prime}, p=p_{b}(z)+p^{\prime}, T_{f}=T_{f b}(z)+T_{f}^{\prime}, T_{s}=T_{s b}(z)+T_{s}^{\prime}, \vec{H}=\vec{H}_{b}(z)+\vec{H}^{\prime} \\
& \vec{M}=\vec{M}_{b}(z)+\vec{M}^{\prime}, \quad \vec{Q}=\vec{Q}_{b}(z)+\vec{Q}^{\prime} .
\end{aligned}
$$

The perturbed quantities, $\vec{q}^{\prime}=\left(u^{\prime}, v^{\prime}, w^{\prime}\right), p^{\prime}, T_{f}^{\prime}, T_{s}^{\prime}, \vec{H}^{\prime}=\left(H_{x}^{\prime}, H_{y}^{\prime}, H_{z}^{\prime}\right), \vec{M}^{\prime}=\left(M_{x}^{\prime}, M_{y}^{\prime}, M_{z}^{\prime}\right)$ and $\vec{Q}^{\prime}=\left(Q_{x}^{\prime}, Q_{y}^{\prime}, Q_{z}^{\prime}\right)$ are assumed to be small. Substituting Eq. (12) into Eqs. (8) and (9) and using Eqs. (5) and (6), we obtain (after dropping the primes)

$$
H_{x}+M_{x}=\left(1+M_{0} / H_{0}\right) H_{x}, H_{y}+M_{y}=\left(1+M_{0} / H_{0}\right) H_{y}, H_{z}+M_{z}=(1+\chi) H_{z}-K T_{f} .
$$

Again substituting Eq. (12) into Eq. (2), linearizing, eliminating the pressure term by taking curl twice and using Eq. (13) the z-component of the resulting equation can be obtained as (after dropping the primes):

$$
0=-\mu_{0} K \beta \frac{\partial}{\partial z}\left(\nabla_{h}^{2} \varphi\right)+\frac{\mu_{0} K^{2} \beta}{1+\chi} \nabla_{h}^{2} T_{f}+\rho_{0} \alpha_{t} g \nabla_{h}^{2} T_{f}
$$

where, $\nabla_{h}^{2}=\partial^{2} / \partial x^{2}+\partial^{2} / \partial y^{2}$ is the horizontal Laplacian operator. Equations (3) and (4), after using Eq. (12) and linearizing, take the following form (after dropping the primes):

$$
\begin{aligned}
& \varepsilon\left(\rho_{0} c\right)_{f} \frac{\partial T_{f}}{\partial t}+\left(\rho_{0} c\right)_{f} w \frac{d T_{f b}}{d z}=\varepsilon k_{f} \nabla^{2} T_{f}+h_{t}\left(T_{s}-T_{f}\right) \\
& (1-\varepsilon)\left(\rho_{0} c\right)_{s} \frac{\partial T_{s}}{\partial t}=-(1-\varepsilon) \nabla \cdot \vec{Q}-h_{t}\left(T_{s}-T_{f}\right) .
\end{aligned}
$$

Equation (7), after substituting Eq. (12), may be written as (after dropping the primes)

$$
\tau_{s} \frac{\partial \vec{Q}}{\partial t}=-\vec{Q}-k_{s} \nabla T_{s}
$$

It is convenient to eliminate $\vec{Q}$ from Eq.(16), using Eq. (17), to get 


$$
(1-\varepsilon)\left(\rho_{0} c\right)_{s}\left(\tau_{s} \frac{\partial}{\partial t}+1\right) \frac{\partial T_{s}}{\partial t}=(1-\varepsilon) k_{s} \nabla^{2} T_{s}-h_{t}\left(\tau_{s} \frac{\partial}{\partial t}+1\right)\left(T_{s}-T_{f}\right)
$$

It is seen that Eq. (18) is effectively hyperbolic and oscillatory instability may be possible with increasing $\tau_{s}$. Equation (18) becomes parabolic when $\tau_{s}=0$. Equations (5) and (6), after substituting Eq. (12) and using Eq. (13), may be written as (after dropping the primes)

$$
\left(1+\frac{M_{0}}{H_{0}}\right) \nabla_{h}^{2} \varphi+(1+\chi) \frac{\partial^{2} \varphi}{\partial z^{2}}-K \frac{\partial T_{f}}{\partial z}=0 .
$$

The normal mode expansion of the dependent variables is assumed in the form $\left\{w, T_{f}, T_{s}, \varphi\right\}=\left\{W(z), \Theta_{f}(z), \Theta_{s}(z), \Phi(z)\right\} \exp [i(\ell x+m y)+\sigma t]$

where, $\ell$ and $m$ are wave numbers in the $x$ and $y$ directions, respectively, $W(z)$ is the amplitude of vertical component of perturbed velocity, $\Theta_{f}(z)$ is the amplitude of perturbed fluid temperature, $\Theta_{s}(z)$ is the amplitude of perturbed solid temperature, $\Phi(z)$ is the amplitude of perturbed magnetic potential and $\sigma$ is the complex growth rate of the disturbance.

Substituting Eq. (20) into Eqs. (14), (15), (18) and (19), and non-dimensionalizing the variables by setting

$$
\begin{aligned}
& \left(x^{*}, y^{*}, z^{*}\right)=\left(\frac{x^{*}}{d}, \frac{y^{*}}{d}, \frac{z^{*}}{d}\right), t^{*}=\frac{\kappa_{f}}{d^{2}} t, W^{*}=\frac{d}{\varepsilon \kappa_{f}} W, \quad \Theta_{f}^{*}=\frac{1}{\beta d} \Theta_{f} \\
& \Theta_{s}^{*}=\frac{1}{\beta d} \Theta_{s}, \Phi^{*}=\frac{(1+\chi)}{\mathrm{K} \beta d^{2}} \Phi
\end{aligned}
$$

where, $\kappa_{f}=k_{f} /\left(\rho_{0} c\right)_{f}$ is the effective thermal diffusivity of the fluid, we obtain the following dimensionless equations (after dropping the asterisks):

$$
\begin{aligned}
& \left(D^{2}-a^{2}\right) W=a^{2} R_{D}\left[M_{1} D \Phi-\left(1+M_{1}\right) \Theta_{f}\right] \\
& {\left[\sigma-\left(D^{2}-a^{2}\right)\right] \Theta_{f}-H_{t}\left(\Theta_{s}-\Theta_{f}\right)=W} \\
& {\left[\alpha \sigma(\tau \sigma+1)-\left(D^{2}-a^{2}\right)\right] \Theta_{s}+\gamma H_{t}(\tau \sigma+1)\left(\Theta_{s}-\Theta_{f}\right)=0} \\
& \left(D^{2}-a^{2} M_{3}\right) \Phi-D \Theta_{f}=0 .
\end{aligned}
$$

Here, $D=d / d z$ is the differential operator, $a=\sqrt{\ell^{2}+m^{2}}$ is the overall horizontal wave number, $R_{D}=\rho_{0} \alpha_{t} g \beta k d^{2} / \varepsilon \mu_{f} \kappa_{f}$ is the Darcy-Rayleigh number and it is a ratio of buoyant to 
viscous forces, $M_{1}=\mu_{0} K^{2} \beta /(1+\chi) \alpha_{t} \rho_{0} g$ is the magnetic number and it is a ratio of magnetic to gravitational forces, $M_{3}=\left(1+M_{0} / H_{0}\right) /(1+\chi)$ is the measure of nonlinearity of magnetization, $H_{t}=h_{t} d^{2} / \varepsilon k_{f}$ is the scaled inter-phase heat transfer coefficient, and $\gamma=\varepsilon k_{f} /(1-\varepsilon) k_{s}$ is the porosity modified conductivity ratio, $\alpha=\left(\rho_{0} c\right)_{s} k_{f} /\left(\rho_{0} c\right)_{f} k_{s}$ is the ratio of conductivities and $\tau=\tau_{s} \kappa_{f} / d^{2}$ is the non-dimensional solid thermal relaxation time parameter.

Equations (22)-(25) are to be solved subject to appropriate boundary conditions. The boundaries are impermeable, ferromagnetic, and perfect thermal conductors. The boundary conditions are:

$W=\Theta_{f}=\Theta_{s}=D \Phi=0 \quad$ at $z=0,1$.

Equations (22)-(25) admit solutions in the form

$W=A_{1} \sin \pi z, \Theta_{f}=A_{2} \sin \pi z, \Theta_{s}=A_{3} \sin \pi z, \Phi=A_{4} \cos \pi z$

where $A_{1}-A_{4}$ are constants. The substitution of Eq. (27) into Eqs. (22)-(25), followed by elimination of the constants $A_{1}-A_{4}$ from the resulting equations, yields the following characteristic equation:

$\left|\begin{array}{cccc}\delta & -a^{2} R_{D}\left(1+M_{1}\right) & 0 & -\pi a^{2} R_{D} M_{1} \\ -1 & \left(\sigma+\delta+H_{t}\right) & -H_{t} & 0 \\ 0 & -\gamma H_{t}(\tau \sigma+1) & \delta+(\tau \sigma+1)\left(\sigma \alpha+\gamma H_{t}\right) & 0 \\ 0 & \pi & 0 & \left(\pi^{2}+M_{3} a^{2}\right)\end{array}\right|=0$

where, $\delta=\pi^{2}+a^{2}$. One of the ways in which Eq. (28) may be used to examine the stability of the system will now be discussed. All the parameters, except the Darcy-Rayleigh number $R_{D}$, are taken as given. Expanding the determinant gives the following expression for $R_{D}$ :

$$
R_{D}=\frac{\left(\pi^{2}+M_{3} a^{2}\right) \delta\left[(\delta+\sigma)\{\delta+\alpha \sigma(\tau \sigma+1)\}+H_{t}\{(\alpha+\gamma) \sigma(\tau \sigma+1)+\delta(1+\gamma+\tau \sigma \gamma)\}\right]}{\left\{\pi^{2}+\left(1+M_{1}\right) M_{3} a^{2}\right\} a^{2}\left(\delta+(\tau \sigma+1)\left(H_{t} \gamma+\alpha \sigma\right)\right)} .
$$


To examine the instability of the system, the real part of $\sigma$ is set to zero and we take $\sigma=i \omega$. Substituting $\sigma=i \omega$ in Eq. (29) and clearing the complex quantities from the denominator, we get

$$
R_{D}=\frac{\left(\pi^{2}+M_{3} a^{2}\right) \delta\left(\Delta_{1}+i \omega \Delta_{2}\right)}{a^{2}\left\{\pi^{2}+\left(1+M_{1}\right) M_{3} a^{2}\right\}\left\{2 H_{t} \gamma \delta+\delta^{2}-2 \tau \alpha \delta \omega^{2}+\left(H_{t}^{2} \gamma^{2}+\alpha^{2} \omega^{2}\right)\left(1+\tau^{2} \omega^{2}\right)\right\}}
$$

where,

$$
\begin{aligned}
\Delta_{1}=H_{t}^{2} \gamma \delta\left(1+\gamma+\tau^{2} \gamma \omega^{2}\right) & +\delta\left\{\delta^{2}-2 \tau \alpha \delta \omega^{2}+\alpha^{2} \omega^{2}\left(1+\tau^{2} \omega^{2}\right)\right\} \\
+ & H_{t}\left\{\delta^{2}+2 \delta^{2} \gamma-2 \tau \alpha \delta \omega^{2}+\alpha^{2} \omega^{2}\left(1+\tau^{2} \omega^{2}\right)\right\}
\end{aligned}
$$

$\Delta_{2}=\delta^{2}+2 H_{t} \gamma \delta-2 \tau \alpha \delta \omega^{2}+\alpha^{2} \omega^{2}\left(1+\tau^{2} \omega^{2}\right)+H_{t}^{2} \gamma\left(\alpha+\gamma-\tau \delta+\tau^{2} \alpha \omega^{2}+\tau^{2} \gamma \omega^{2}\right)$.

Since the Rayleigh number $R_{D}$ is a physical quantity, it must be real. Hence, from Eq. (30) it implies either $\omega=0$ or $\Delta_{2}=0(\omega \neq 0)$, and accordingly the condition for the onset of stationary and oscillatory convection is obtained.

\subsection{Stationary convection $(\omega=0)$}

The stationary convection (direct bifurcation) corresponds to $\omega=0$ and it occurs at $R_{D}=R_{D}^{s}$, where

$$
R_{D}^{s}=\frac{\left(\pi^{2}+M_{3} a^{2}\right) \delta^{2}\left\{H_{t}(1+\gamma)+\delta\right\}}{a^{2}\left\{\pi^{2}+\left(1+M_{1}\right) M_{3} a^{2}\right\}\left(\delta+\gamma H_{t}\right)} .
$$

Equation (31) reveals that the Cattaneo effects have no influence on the onset of stationary convection because $\tau$ is not appearing in the expression for $R_{D}^{s}$. This is because the basic state remains the same irrespective of the Cattaneo effect as it corresponds to pure conduction. Equation (31) coincides with the expression obtained by Lee et al. [21]. We note that $R_{D}^{s}$ attains its critical value $R_{D c}^{s}$ at $a^{2}=a_{c}^{2}$, where $a_{c}^{2}$ satisfies the equation

$$
b_{1}\left(a_{c}^{2}\right)^{6}+b_{2}\left(a_{c}^{2}\right)^{5}+b_{3}\left(a_{c}^{2}\right)^{4}+b_{4}\left(a_{c}^{2}\right)^{3}+b_{5}\left(a_{c}^{2}\right)^{2}+b_{6}\left(a_{c}^{2}\right)+b_{7}=0
$$

where,

$$
\begin{aligned}
& b_{1}=\left(1+M_{1}\right) M_{3}^{2} \\
& b_{2}=2 M_{3}\left\{\left(1+M_{3}+M_{1} M_{3}\right) \pi^{2}+H_{t}\left(1+M_{1}\right) M_{3} \gamma\right\}
\end{aligned}
$$




$$
\begin{aligned}
& b_{3}=\left\{1-2\left(-2+M_{1}\right) M_{3}\right\} \pi^{4}+H_{t}^{2}\left(1+M_{1}\right) M_{3}^{2} \gamma(1+\gamma) \\
& +H_{t} M_{3} \pi^{2}\left\{-M_{3}+4 \gamma+2 M_{3} \gamma+M_{1}\left(-1-M_{3}+2 M_{3} \gamma\right)\right\} \\
& b_{4}=-2 \pi^{2}\left[\begin{array}{r}
\left\{-1+M_{3}^{2}+M_{1} M_{3}\left(3+M_{3}\right)\right\} \pi^{4}-H_{t}^{2} M_{3} \gamma(1+\gamma) \\
+H_{t} \pi^{2}\left\{-\gamma+\left(1+M_{1}\right) M_{3}^{2}(1+\gamma)+M_{3}\left(1-2 \gamma+2 M_{1}(1+\gamma)\right)\right\}
\end{array}\right] \\
& b_{5}=-\pi^{4}\left[\begin{array}{r}
M_{3}\left\{4+M_{3}+M_{1}\left(6+M_{3}\right)\right\} \pi^{4}+H_{t}^{2}\left\{-1+M_{3}^{2}+M_{1} M_{3}\left(2+M_{3}\right)\right\} \gamma(1+\gamma) \\
+H_{t} \pi^{2}\left\{1-2 \gamma+\left(1+M_{1}\right) M_{3}^{2}(1+2 \gamma)+M_{3}\left(4+5 M_{1}+4 \gamma+8 M_{1} \gamma\right)\right\}
\end{array}\right] \\
& b_{6}=-2 \pi^{6}\left(H_{t}+\pi^{2}+H_{t} \gamma\right)\left\{\left(1+M_{3}+M_{1} M_{3}\right) \pi^{2}+H_{t}\left(1+M_{1}\right) M_{3} \gamma\right\} \\
& b_{7}=-\pi^{8}\left\{\pi^{4}+H_{t}^{2} \gamma(1+\gamma)+H_{t} \pi^{2}(1+2 \gamma)\right\} \text {. }
\end{aligned}
$$

Equation (32) is solved numerically for various values of $\gamma, M_{1}, M_{3}$ and $H_{t}$ the critical value of $a_{c}^{2}$ is obtained. Using this $a_{c}^{2}$ in Eq. (31), the critical Rayleigh number $R_{D c}^{s}$ is obtained, above which the convection sets in. It is interesting to check Eq. (31) under some limiting cases. When $M_{1}=0$ (for ordinary fluid), Eq. (31) reduces to

$$
R_{D}^{s}=\frac{\delta^{2}\left\{H_{t}(1+\gamma)+\delta\right\}}{a^{2}\left(\delta+\gamma H_{t}\right)}
$$

The above expression is the same as the one obtained by Banu and Rees [35] and Straughan [29]. When $H_{t}=0$, Eq. (33) simply reduces to

$$
R_{D}^{s}=\frac{\delta^{2}}{a^{2}}
$$

Evidently, $R_{D}^{s}$ given by Eq.(34) attains its minimum at $a=\pi$. That is, the critical DarcyRayleigh number is $4 \pi^{2}$ and the associated critical wave number is $\pi$; the known results for the classical Darcy-Bénard problem.

\subsection{Oscillatory convection $(\omega \neq 0)$}

The oscillatory onset corresponds to $\Delta_{2}=0(\omega \neq 0)$ in Eq. (30) and this gives a dispersion relation of the form

$$
B_{1}\left(\omega^{2}\right)^{2}+B_{2}\left(\omega^{2}\right)+B_{3}=0
$$

where,

$$
\begin{aligned}
& B_{1}=\tau^{2} \alpha^{2} \\
& B_{2}=\alpha^{2}+H_{t}^{2} \tau^{2} \gamma^{2}+\tau \alpha\left(H_{t}^{2} \tau \gamma-2 \delta\right)
\end{aligned}
$$




$$
B_{3}=2 H_{t} \gamma \delta+\delta^{2}+H_{t}^{2} \gamma(\gamma+\alpha-\tau \delta) \text {. }
$$

For the occurrence of oscillatory convection, $\omega^{2}$ should be positive. From Eq. (35), it is observed that the coefficient $B_{1}$ is always positive while the coefficients $B_{2}$ and/or $B_{3}$ can be negative depending on the choices of parametric values. If this is the case, then from Descartes' rule of signs it follows that one or two positive values of $\omega^{2}$ exist indicating the occurrence of oscillatory convection for those choices of parametric values. However, if $\tau=0$ (i.e. in the absence of Cattaneo effect in the solid) then it is noted that both $B_{2}$ and $B_{3}$ are positive and the occurrence of oscillatory convection is ruled out. Thus, it is evident that the Cattaneo effect in the solid is the cause to initiate the occurrence of convective instability via oscillatory convection.

The oscillatory convection occurs at $R_{D}=R_{D}^{o}$, where

$$
R_{D}^{o}=\frac{\left(\pi^{2}+M_{3} a^{2}\right) \delta \Delta_{1}}{a^{2}\left\{\pi^{2}+\left(1+M_{1}\right) M_{3} a^{2}\right\}\left\{2 H_{t} \gamma \delta+\delta^{2}-2 \tau \alpha \delta \omega^{2}+\left(H_{t}^{2} \gamma^{2}+\alpha^{2} \omega^{2}\right)\left(1+\tau^{2} \omega^{2}\right)\right\}}
$$

and $\omega^{2}$ is given by Eq.(35). When $M_{1}=0$ or $M_{3}=0$, Eq. (36) reduces to the case of ordinary viscous fluid. The critical oscillatory Darcy-Rayleigh number $R_{D c}^{o}$ with respect to the wave number is computed numerically as follows. Equation (35) is solved first to determine the positive values of $\omega^{2}$ for chosen parametric values. If there are none, then no oscillatory convection is possible. If there is only one positive value of $\omega^{2}$ then the critical value of $R_{D}^{o}$ with respect to the wave number is computed numerically from Eq. (36). If there are two positive values of $\omega^{2}$ then there corresponds a value of $R_{D}^{o}$ for each positive value of $\omega^{2}$. That is, oscillatory convection sets in simultaneously at two different frequencies but for the same wave number and in which case closed oscillatory neutral curve may occur. Since we are dealing with convective instability problem, we are interested in the least value of Rayleigh number at which the oscillatory convection sets in first. Therefore, the lower value of $R_{D}^{o}$ is preferred for each fixed wave number and the critical value of this with respect to the wave number is computed.

\section{Results and discussion}

The local thermal non-equilibrium (LTNE) model is used with Cattaneo effects in the solid to investigate the onset of ferroconvection in a horizontal layer of Darcy porous medium. The range of values of magnetic parameters chosen are based on the physical parameters for a commercially 
available magnetic fluid EMG 905 produced by Ferrofluidics (Auernhammer and Brand [36]); density $\rho\left[\mathrm{kg} / \mathrm{m}^{3}\right]=1.24 \times 10^{3}$, kinematic viscosity $\left(27^{0} \mathrm{C}\right) v\left[\mathrm{~m}^{2} / \mathrm{s}\right]=12 \times 10^{-6}$, thermal diffusivity $\kappa\left[\mathrm{m}^{2} / \mathrm{s}\right]=8 \times 10^{-8}$, heat capacity $c[\mathrm{~J} / \mathrm{kgK}]=1.47 \times 10^{3}$, coefficient of thermal expansion $\alpha_{t}[1 / K]=8.6 \times 10^{-4}$, susceptibility at low field $\chi=1.9$, pyromagnetic coefficient at $H=50 \mathrm{kA} / \mathrm{m}[\mathrm{A} / \mathrm{Km}]=110$ and mean particle diameter $[\mathrm{nm}]=10.2$. For such fluids, the magnetic parameters have the following order of magnitude: $M_{1} \sim 10^{-4}-10$ and $M_{3} \geq 1$. In addition, the results are also presented for copper oxide and aluminium oxide solid skeletons as both materials have practical use in heat exchangers.

The typical steady and oscillatory neutral stability curves in the $\left(R_{D}, a^{2}\right)$-plane for different values of $\tau$ (with $\alpha=1$ ) and $\alpha$ (with $\tau=0.5$ ) are shown in Figs. 2(a) and (b), respectively when $\gamma=1, M_{1}=1, M_{3}=1$ and $H_{t}=15$. The figures show the upward concave shape of the neutral stability curves, typical of stability problems of the Darcy-Bénard type, and the unstable state lies above each neutral stability curve. From the figures it is evident that increasing $\tau$ is to decrease the region of stability while an opposite trend is noticed with increasing $\alpha$. Further inspection of Fig. 2(a) reveals that there exists a threshold value of $\tau$ beyond which only oscillatory convection is preferred and for the present case it is 0.3258 . Whereas Fig. 2(b) shows that oscillatory convection is preferred for values of $\alpha$ lower than 5.55. Thus the presence of Cattaneo effect in the solid is to instigate the occurrence of convective instability via oscillatory motions under certain conditions, whereas in the absence of such effects the instability occurs always via stationary convection (Lee et al. [21]).

The critical Darcy-Rayleigh number for the onset of stationary $\left(R_{D c}^{s}\right)$ and oscillatory $\left(R_{D c}^{O}\right)$ convection, the corresponding critical wave number $\left(a_{c}^{2}\right)$ and the critical frequency of oscillation $\left(\omega_{c}^{2}\right)$ are computed numerically for various values of physical parameters and the results are summarized in Figs. 3-7. Figures 3(a), (b) and (c) show the variation of critical DarcyRayleigh numbers, the critical wave number and the critical frequency of oscillation, respectively as a function of $H_{t}$ for different values of $\tau\left(=0.5,1\right.$ and 10) and for two values of $M_{1}(=0$ and 0.6 ) with $M_{3}=1, \alpha=1$ and $\gamma=1$. Figure 3(a) shows that, depending on the parametric values, the instability occurs via oscillatory convection when $H_{t}$ exceeds a threshold value $H_{t}^{T}$, and this 
value decreases significantly with increasing $\tau$. It is noted that the onset of stationary convection (denoted by s) is delayed, while the onset of oscillatory convection (denoted by o) is hastened with increasing $H_{t}$. Thus the effect of inter-phase heat-transfer coefficient $H_{t}$ on the nature of convective instability is not consistent. Moreover, the influence of $H_{t}$ on the oscillatory onset is more noticeable in the beginning and thereafter its effect becomes fairly insensitive. This behavior is found to be more pronounced when $\tau=10$. The size of $M_{1}$ is related to the importance of magnetic forces compared to gravitational forces. The case $M_{1}=0$ corresponds to convective instability in an ordinary viscous fluid saturating a porous medium. It is observed that an increase in $M_{1}$ is to decrease both the stationary and oscillatory critical Rayleigh numbers suggesting the ferrofluid carries heat more efficiently than the ordinary viscous fluid as it hastens the onset of ferroconvection. This is due to an increase in the destabilizing magnetic force with increasing $M_{1}$, which favours the fluid to flow more easily. Also the threshold value $H_{t}^{T}$, beyond which oscillatory convection is preferred, increases with increasing $M_{1}$.

The variation of critical wave number as a function of $H_{t}$ is exhibited in Fig. 3(b). It is seen that the critical wave number for the oscillatory convection (denoted by o) starts beyond threshold value $H_{t}^{T}$ for each case. The values of $a_{c}^{2}$ increase sharply with $H_{t}$ initially and become almost insensitive with further increase in $H_{t}$ and this trend is more predominant at $\tau=10$. Thus the size of convection cells decreases with increasing $H_{t}$. It is further seen that increasing $M_{1}$ is to increase, while increasing $\tau$ is to decrease $a_{c}^{2}$ and hence their effect is to decrease and enlarge the size of convection cells, respectively. It is also observed that the values of $a_{c}^{2}$ for stationary convection are higher than those of oscillatory convection.

The critical frequency of oscillations $\omega_{c}^{2}$ displayed in Fig. 3(c) shows that the variation in $\omega_{c}^{2}$ with $H_{t}$ is significant for $\tau=0.5$ and 1 , while this trend is found to be not so high significant for $\tau=10$. However, the parameter $M_{1}$ exhibits a dual effect on $\omega_{c}^{2}$ with increasing $H_{t}$. It is observed that, initially, increase in $M_{1}$ is to decrease $\omega_{c}^{2}$ up to a certain value of $H_{t}$, and thereafter an opposite behavior could be seen. 
The effect of non linearity of magnetization parameter $M_{3}$ on the stability characteristics of the system is presented in Figs. 4(a-c) for two values $M_{3}\left(=1\right.$ and 10) as a function of $H_{t}$ with $M_{1}=0.6, \alpha=1$ and $\gamma=1$. From Fig. 4(a), it is noted that both onset of stationary and oscillatory convection is hastened with increasing $M_{3}$. Increase in the value of $M_{3}$ makes the ferrofluid to acquire larger magnetization which in turn interacts with the imposed magnetic field and releases more energy to drive the flow faster. Alternatively, a higher value of $M_{3}$ would arise either due to a larger pyromagnetic coefficient or larger temperature gradient. Both these factors are conducive for generating a larger gradient in the Kelvin body force field, possibly promoting the instability. Further, it is seen that oscillatory convection is preferred at decreasing threshold values of $H_{t}$ with increasing $M_{3}$. It is seen that increasing $M_{3}$ is to decrease $a_{c}^{2}$ and its effect is to increase the size of convection cells (Fig. 4(b)). Figure 4(c) shows that the parameter $M_{3}$ exhibits a dual effect on $\omega_{c}^{2}$ with increasing $H_{t}$. Initially, increase in $M_{3}$ is to increase $\omega_{c}^{2}$ up to a certain value of $H_{t}$, and thereafter the trend gets reversed.

The impact of conductivity ratio $\alpha$ on the stability characteristics of the system is displayed in Figs. 5(a-c) for two values of $\alpha$ as a function of $H_{t}$ for selected values of $\tau$ with $M_{1}=0.6, M_{3}=1$ and $\gamma=1$. From the figures, it is seen that the effect of increasing $\alpha$ is to delay the onset of oscillatory convection and thereby to increase the threshold value of $H_{t}$ (Fig. 5a). Also, the effect of $\alpha$ on the onset of oscillatory convection diminishes with increasing $\tau$. The critical wave number increases with $\alpha$ indicating its effect is to decrease the size of convection cells (Fig. 5b). For a fixed value of $\tau, \omega_{c}^{2}$ decreases with increasing $\alpha$ (Fig. 5c).

The values of $\alpha$ and $\gamma$ for copper oxide and aluminium oxide solid skeletons are given by (Straughan [29] and references therein)

$$
\alpha_{\text {CuO }}=8.664 \times 10^{-4}, \gamma_{\text {CuO }}=6.403 \times 10^{-4} \text { and } \alpha_{\mathrm{Al}_{2} \mathrm{O}_{3}}=1.420 \times 10^{-2}, \gamma_{\mathrm{Al}_{2} \mathrm{O}_{3}}=7.337 \times 10^{-3} \text {. }
$$

For these two sets of parametric values, the results are displayed in Figs. 6 and 7 for two values of $\tau(=10,12)$ with $M_{1}=0.5$ and $M_{3}=1$. It is seen that oscillatory convection is preferred at relatively high values of $H_{t}$ when compared to values of $\alpha=1$ and $\gamma=1$ (see Figs. 3 and 4). This demonstrates the interplay of Cattaneo effects in the solid and various physical parameters on the occurrence of oscillatory convection. As expected, increase in the value of $\tau$ is to hasten the 
onset of oscillatory ferroconvection (Figs. 6a and 7a). Here, it is seen that the critical wave number increases with increasing $H_{t}$ and $\tau$. That is, the size of the convection cells decreases in the $(x, y)$ - plane. The critical wave number of oscillatory convection is lower than that of stationary convection for values of $\tau$ considered indicating the cell size changes by a large amount to a much wider cell (Figs. $6 \mathrm{~b}$ and $7 \mathrm{~b}$ ). The variation of $\omega_{c}^{2}$ with $H_{t}$ is illustrated in Figs. 6(c) and 7(c). From the figures it is observed that increasing $H_{t}$ is to increase $\omega_{c}^{2}$. Nonetheless, the parameter $\tau$ exhibits a dual effect on $\omega_{c}^{2}$ with increasing $H_{t}$. Table 1 shows the transition values from stationary to oscillatory convection observed for different values of solid thermal relaxation time parameter $\tau$ and magnetic number $M_{1}$ when $M_{3}=1, \alpha=1$ and $\gamma=1$. It is seen that the pattern of behavior observed for porous skeletons made of copper oxide $(\mathrm{CuO})$ and aluminium oxide $\left(\mathrm{Al}_{2} \mathrm{O}_{3}\right)$ is reflected in the table (cf. Figs. 6 and 7).

A regime of local thermal non-equilibrium (LTNE) is considered.

\section{Conclusions}

A regime of local thermal non-equilibrium (LTNE) is considered with Cattaneo effect in the solid on the onset of ferroconvection in a horizontal layer of Darcy porous medium. As a result of temperature waves in the solid via a Cattaneo-like heat flux theory, novel consequences have been discovered. If the solid thermal relaxation time $\tau$ exceeds a threshold value, then the instability occurs via oscillatory convection. This is in contrast to the observed instability phenomenon in the absence of Cattaneo effects wherein ferroconvection occurs only via stationary convection. The effect of increasing $\tau$ and decrease in the ratio of diffusivities $\alpha$ is to hasten the onset of oscillatory convection. Increase in the value of inter-phase heat transfer coefficient $H_{t}$ is to delay the onset of stationary convection, while it exhibits an opposite trend on the onset of oscillatory convection. Besides, the threshold value $H_{t}^{T}$ (i.e. the value of $H_{t}$ at which the transition from stationary to oscillatory convection takes place) decreases with increasing $\tau$ noticeably and marginally with increasing $M_{3}$, whereas it increases with increasing $\alpha$ and $M_{1}$. The effect of increasing magnetic parameters $M_{1}$ and $M_{3}$ is to hasten the onset of both stationary and oscillatory ferroconvection. The size of convection cells is enlarged with increasing $M_{3}$ and $\tau$, but it is narrowed with increasing $\alpha$ and $M_{1}$. Moreover, the critical wave number for 
stationary convection is higher than those of oscillatory convection. The results obtained for copper oxide and aluminium oxide solid skeletons also found to exhibit similar behavior.

\section{References}

1. Rosensweig, R.E.: Ferrohydrodynamics. Cambridge University Press, Cambridge, London (1985)

2. Berkovsky, B.M., Medvedev, V.F., Krakov, M.S.: Magnetic Fluids: Engineering Applications. Oxford University Press, New York (1993)

3. Blums, E.S., Cebers, A.O., Maiorov, M.M.: Magnetic Fluids. de Gruyter, New York (1997)

4. Hergt, R., Andrä, W., Ambly, C.G., Hilger, I., Kaiser, W.A., Richter, U., Schmidt, H.G.: Physical limitations of hypothermia using magnetite fine particles. IEEE Trans. Magn. 34, 3745-3754 (1998)

5. Alexiou, C., Arnold, W., Hulin, P., Klein, R., Schmidt, A., Bergemann, C., Parak, F.G.: Therapeutic efficacy of ferrofluid bound anticancer agent. Magnetohydrodynamics. 37, 318 $322(2001)$

6. Odenbach, S.: Recent progress in magnetic fluid research. J. Phys. Condens. Matter. 16, R1135-R1150 (2004)

7. Ganguly, R. Sen, S. Puri, I.K.: Heat transfer augmentation using a magnetic fluid under the influence of a line dipole. J. Magn. Magn. Mater. 271, 63-73 (2004)

8. Kaloni, P.N., Lou, J.X.: Convective instability of magnetic fluids. Phys. Rev. E. 70, 026313$026324(2004)$

9. Nkurikiyimfura, I., Wang, Y., Pan, Z.: Heat transfer enhancement by magnetic nanofluids A review. Renewable and Sustainable Energy Rev. 21, 548-561 (2013)

10. Rosensweig, R.E., Zahn, M., Vogler, T.: Stabilization of fluid penetration through a porous medium using magnetizable fluids. In: Berkovsky B (ed.) Thermomechanics of magnetic fluids. Hemisphere Washington DC 195-211 (1978)

11. Zhan, M., Rosensweig, R.E.: Stability of magnetic fluid penetration through a porous medium with uniform magnetic field oblique to the interface. IEEE Transactions of Mag. MAG-16, 275-282 (1980)

12. Vaidyanathan, G., Sekar, R., Balasubramanian, R.: Ferroconvective instability of fluids saturating a porous medium. Int. J. Engg. Sci. 29, 259-1267 (1991)

13. Qin, Y., Chadam, J.: A non-linear stability problem for ferromagnetic fluids in a porous medium. Appl. Math. Letters. 8(2), 25-29 (1995) 
14. Borglin, S.E., Mordis, J., Oldenburg, C.M.: Experimental studies of the flow of ferrofluid in porous media. Transp. Porous Med. 41, 61-80 (2000)

15. Sunil, Amit Mahajan: A Nonlinear stability analysis for thermoconvective magnetized ferrofluid saturating a porous medium. Transp. Porous Med. 76, 327-343 (2009)

16. Shivakumara, I.S., Nanjundappa, C.E., Ravisha, M.: Thermomagnetic convection in a magnetic nanofluid saturated porous medium. Int. J. Appl. Math. Engg. Sci. 2(2), 157-170 (2008)

17. Shivakumara, I.S., Nanjundappa, C.E., Ravisha, M.: Effect of boundary conditions on the onset of thermomagnetic convection in a ferrofluid saturated porous medium. ASME J. Heat Transf. 131, 101003-1-101003-9 (2009)

18. Nanjundappa, C.E., Shivakumara, I.S., Ravisha, M.: The onset of ferroconvection in a horizontal saturated porous layer heated from below and cooled from above with constant heat flux subject to MFD viscosity. Int. Comm. Heat Mass Transf. 37, 1246-1250 (2010)

19. Virto, L., Carbonell, M., Castilla, R., Gamez-Montero, P.J.: Heating of saturated porous media in practice: several causes of local thermal non-equilibrium. Int. J. Heat Mass Transf. 52, 5412-5422 (2009)

20. Sunil, Poonam Sharma, Amit Mahajan.: Nonlinear ferroconvection in a porous layer using a thermal nonequilibrium model. Special Topics Rev. Porous Med. 1 105-121 (2010)

21. Lee, J., Shivakumara, I.S. Ravisha, M.: Effect of thermal non-equilibrium on convective instability in a ferromagnetic fluid saturated porous medium. Transp. Porous Med. 86, 103124. (2011)

22. Shivakumara, I.S., Lee, J., Ravisha, M., Gangadhara Reddy, R.: The onset of Brinkman ferroconvection using a thermal non-equilibrium model. Int. J. Heat Mass Transf. 54, 2116$2125(2011)$

23. Shivakumara, I.S., Lee, J., Ravisha, M., Gangadhara Reddy, R.: The effects of local thermal nonequilibrium and MFD viscosity on the onset of Brinkman ferroconvection. Meccanica. 47, 1359-1378 (2012)

24. Cattaneo, C.: Sulla conduzione del calore. Atti Sem. Mat. Fis. Univ. Modena 3, 83-101 (1948)

25. Straughan, B., Franchi, F.: Bénard convection and the Cattaneo law of heat conduction. Proc. Roy. Soc. A 96, 175-178 (1984)

26. Lebon, G., Cloot, A.: Bénard-Marangoni, instability in a Maxwell-Cattaneo fluid. Phys. Lett. A 105, 361-364 (1984)

27. Straughan, B.: Thermal convection with the Cattaneo-Christov model. Int. J. Heat Mass Transf. 53, 95-98 (2010) 
28. Straughan, B.: Heat waves, Series in Applied Math Science. Springer, New York 177, (2011)

29. Straughan, B.: Porous convection with local thermal non-equilibrium temperatures and with Cattaneo effects in the solid. Proc. Roy. Soc. A 469, 20130187 (2013)

30. Stranges, D.F., Khayat R.E., Albaalbaki, B.: Thermal convection of non-Fourier fluids. Linear stability. Int. J. Thermal Sci. 74, 14-23 (2014)

31. Haddad, S.A.M.: Thermal instability in Brinkman porous media with Cattaneo-Christov heat flux. Int. J. Heat Mass Transf. 68, 659-668 (2014)

32. Shivakumara, I.S., Ravisha, M., Ng, C.O., Varun, V.L.: A thermal non-equilibrium model with Cattaneo effect for convection in a Brinkman porous layer. Int. J. Non-Linear Mech. 71, 39-47 (2015)

33. Nield, D.A., Bejan, A.: Convection in porous media, 4th Ed., Springer, New York (2013)

34. Finlayson, B.A.: Convective instability of ferromagnetic fluids. J. Fluid Mech. 40, 753-767 (1970)

35. Banu, N., Rees, D.A.S.: Onset of Darcy-Benard convection using a thermal non-equilibrium Model. Int. J. Heat Mass Transf. 45, 2221-2228 (2002)

36. Auernhammer, G. K., Brand, H.R.: Thermal convection in a rotating layer of a magnetic fluid. Eur. Phys. J. B 16, 157-168 (2000)

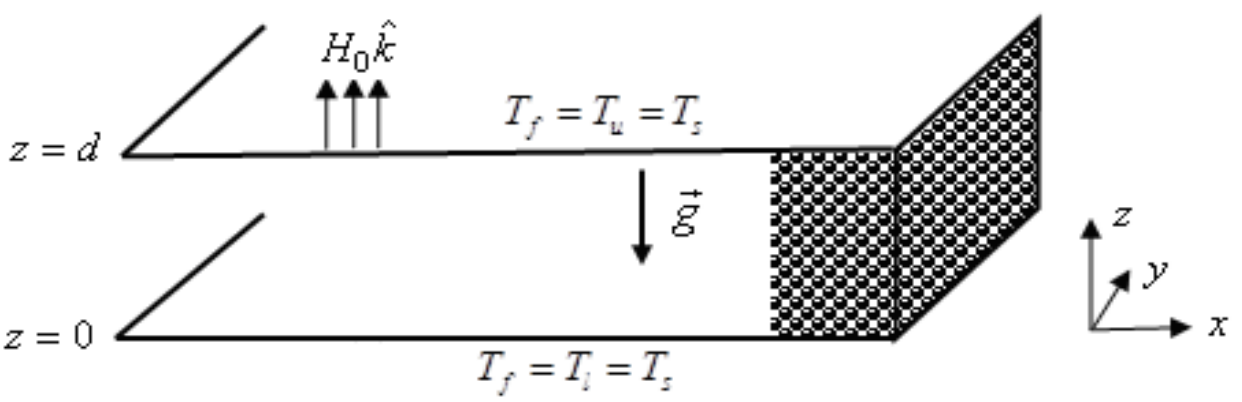

Fig. 1 Physical configuration 

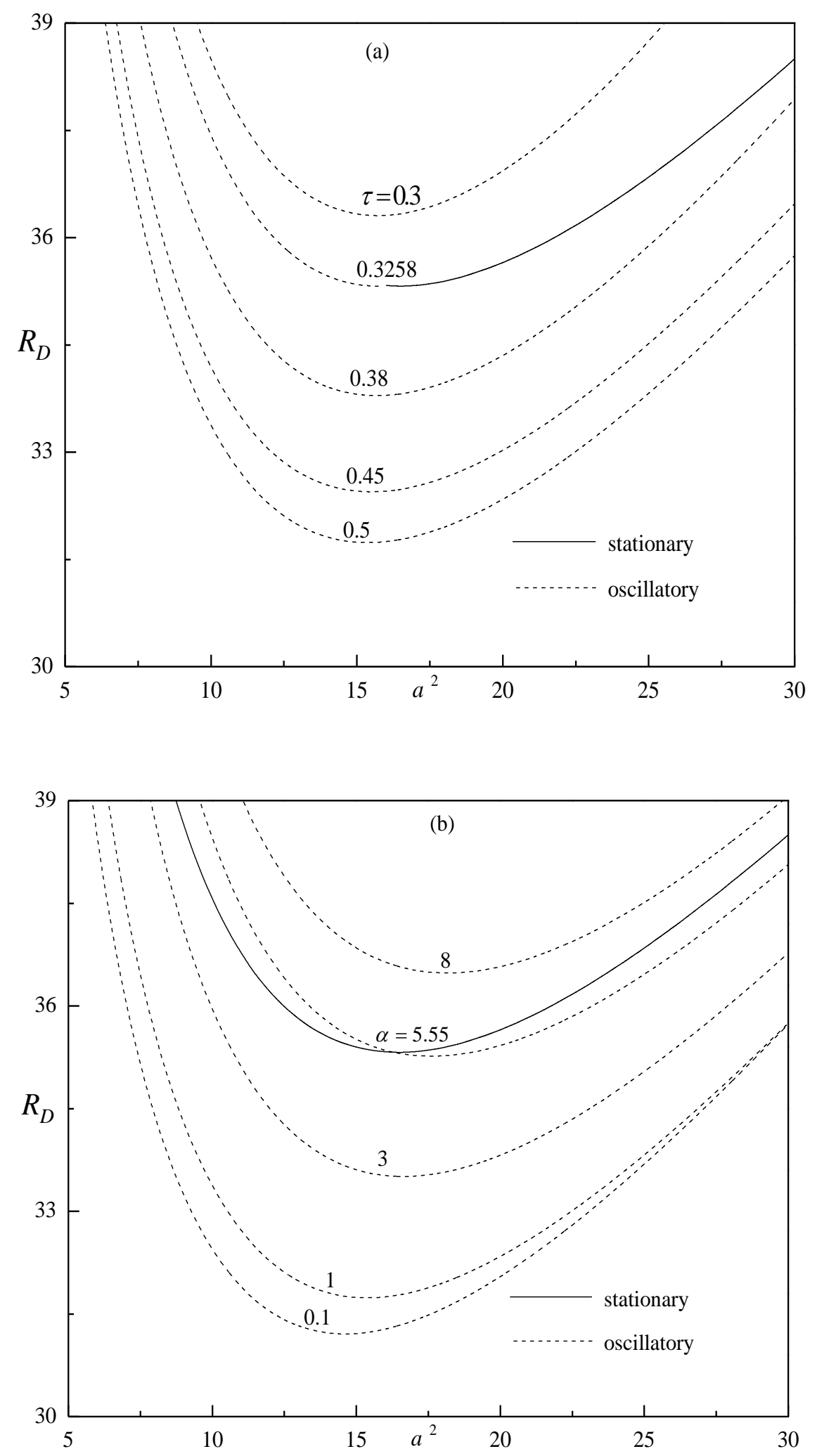

Fig. 2 Neutral curves for different values of (a) $\tau$ with $\alpha=1$ and (b) $\alpha$ with $\tau=0.5$ when $\gamma=1$, $M_{1}=1, M_{3}=1$ and $H_{t}=15$. 

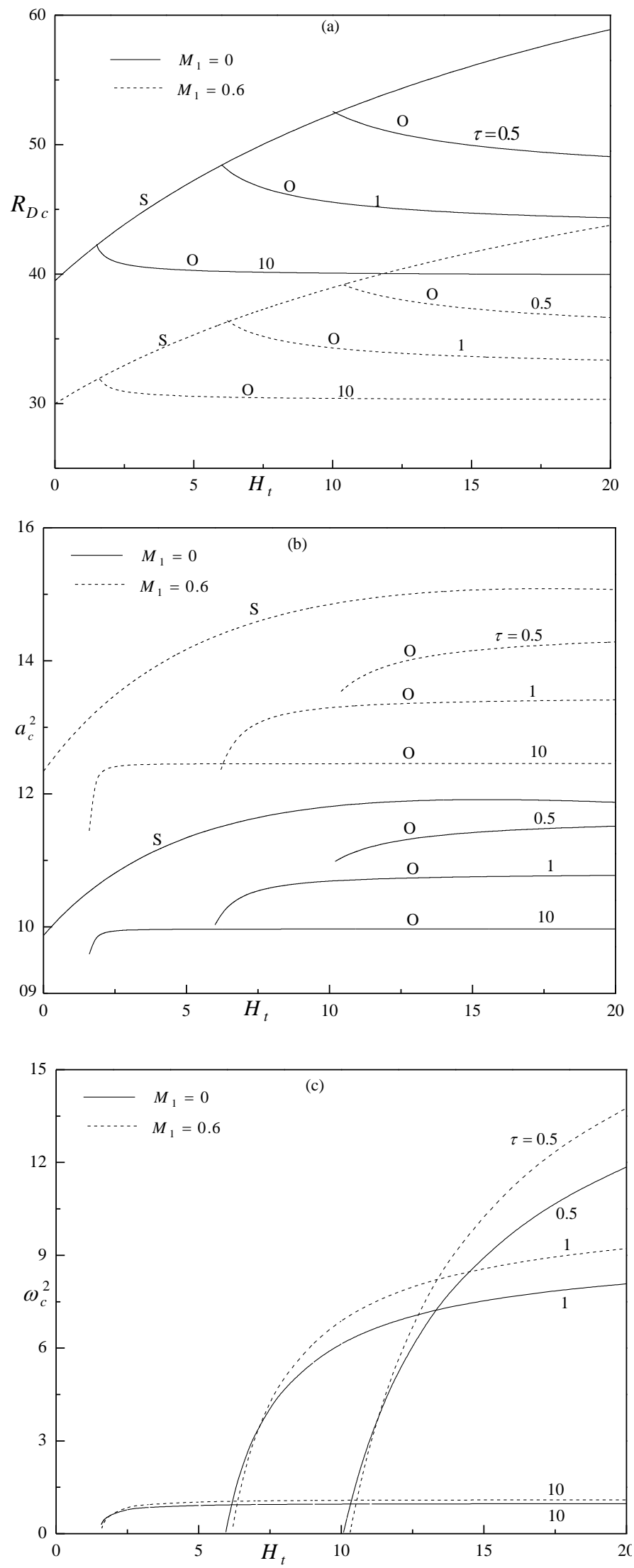

Fig. 3 Variation of (a) $R_{D c}$, (b) $a_{c}^{2}$ (c) $\omega_{c}^{2}$ with $H_{t}$ for two values of $M_{1}$ and three values of $\tau$ with $\gamma=1, M_{3}=1$ and $\alpha=1$. 

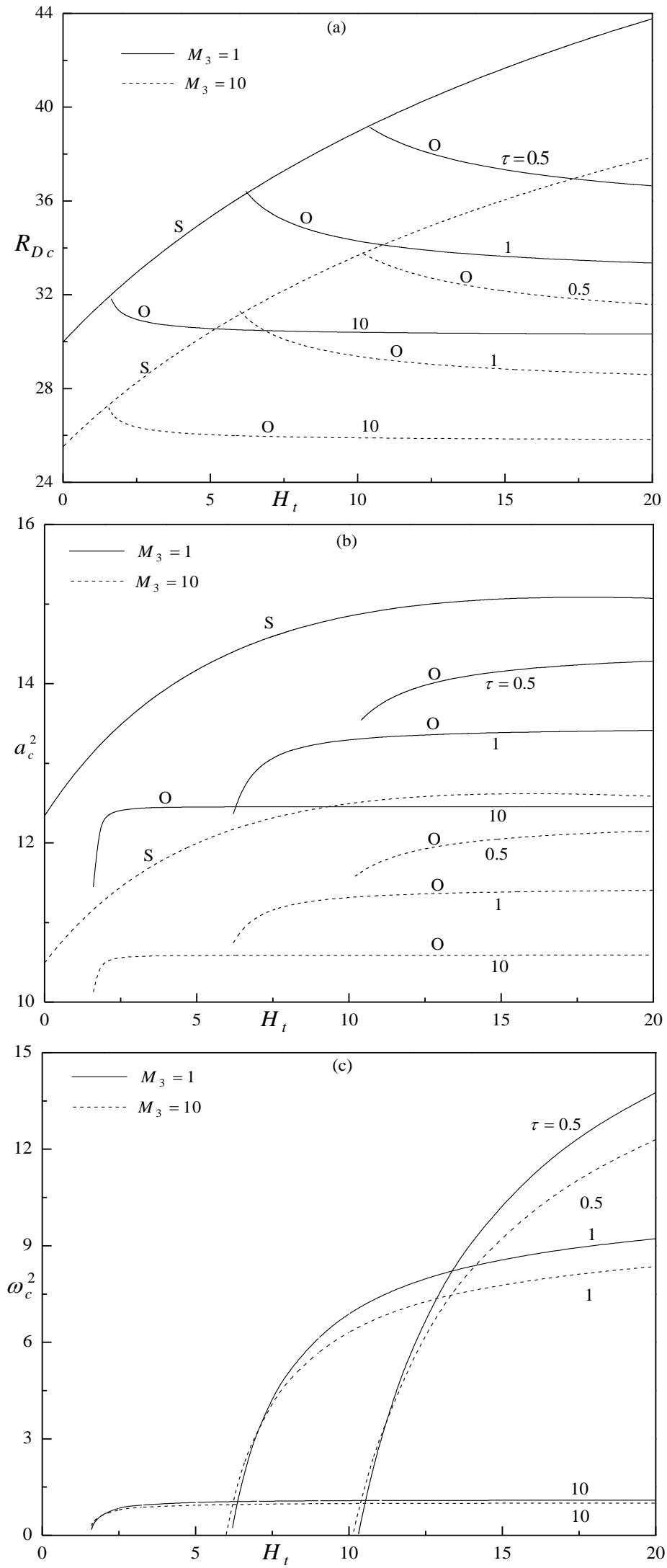

Fig. 4 Variation of (a) $R_{D c}$, (b) $a_{c}^{2}$ (c) $\omega_{c}^{2}$ with $H_{t}$ for two values of $M_{3}$ and three values of $\tau$ with $\gamma=1, M_{1}=0.6$ and $\alpha=1$. 

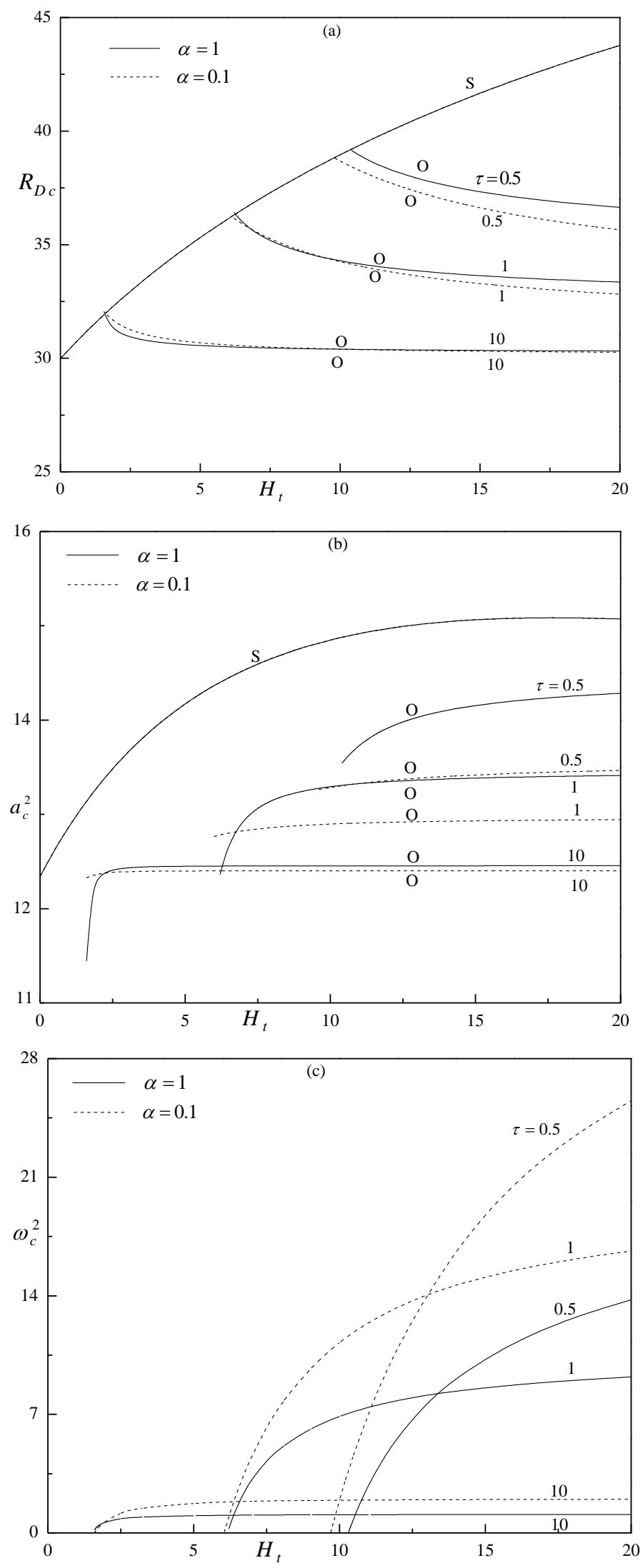

Fig. 5 Variation of (a) $R_{D c}$, (b) $a_{c}^{2}$ (c) $\omega_{c}^{2}$ with $H_{t}$ for two values of $\alpha$ and three values of $\tau$ with $\gamma=1, M_{1}=0.6$ and $M_{3}=1$. 

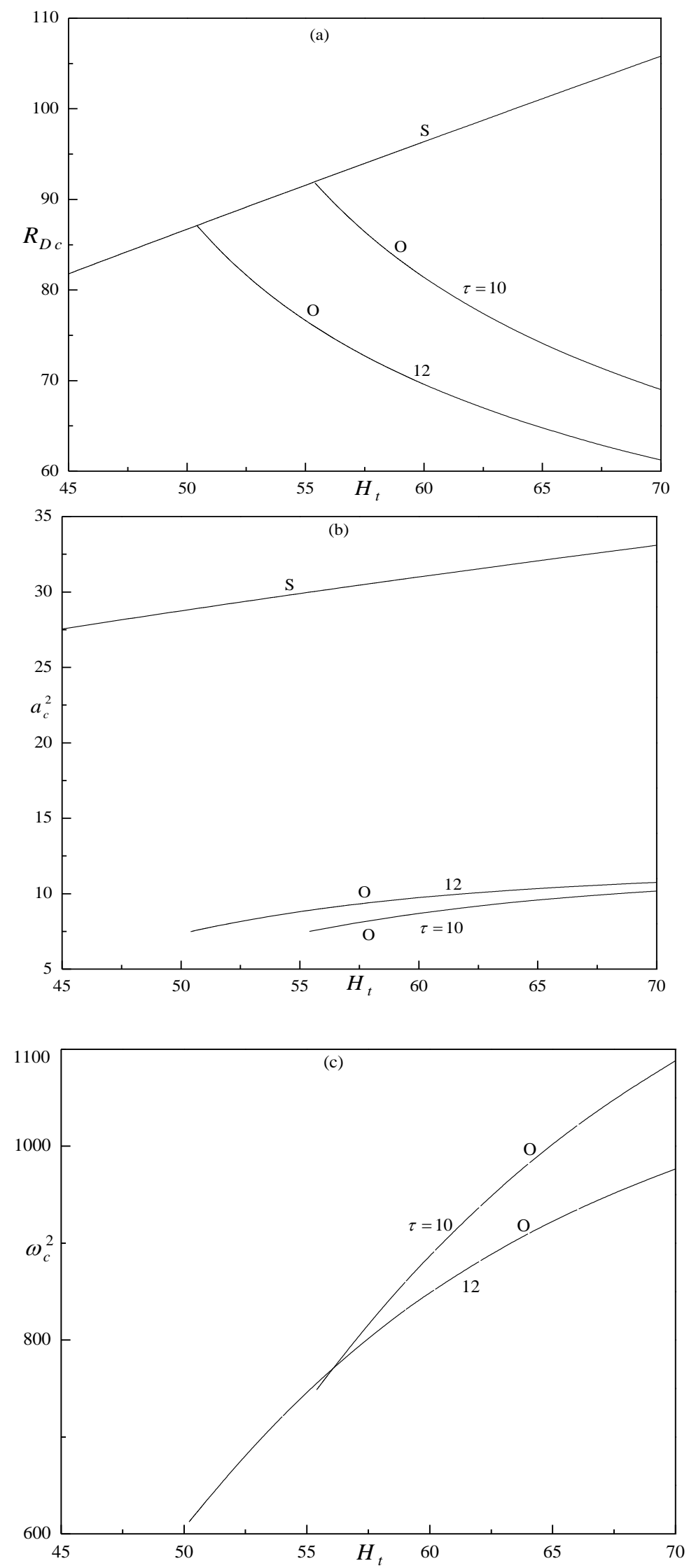

Fig. 6 Variation of (a) $R_{D c}$, (b) $a_{c}^{2}$ (c) $\omega_{c}^{2}$ with $H_{t}$, porous skeleton made of $C u O$ for two values of $\tau$ with $M_{1}=0.5$ and $M_{3}=1$. 

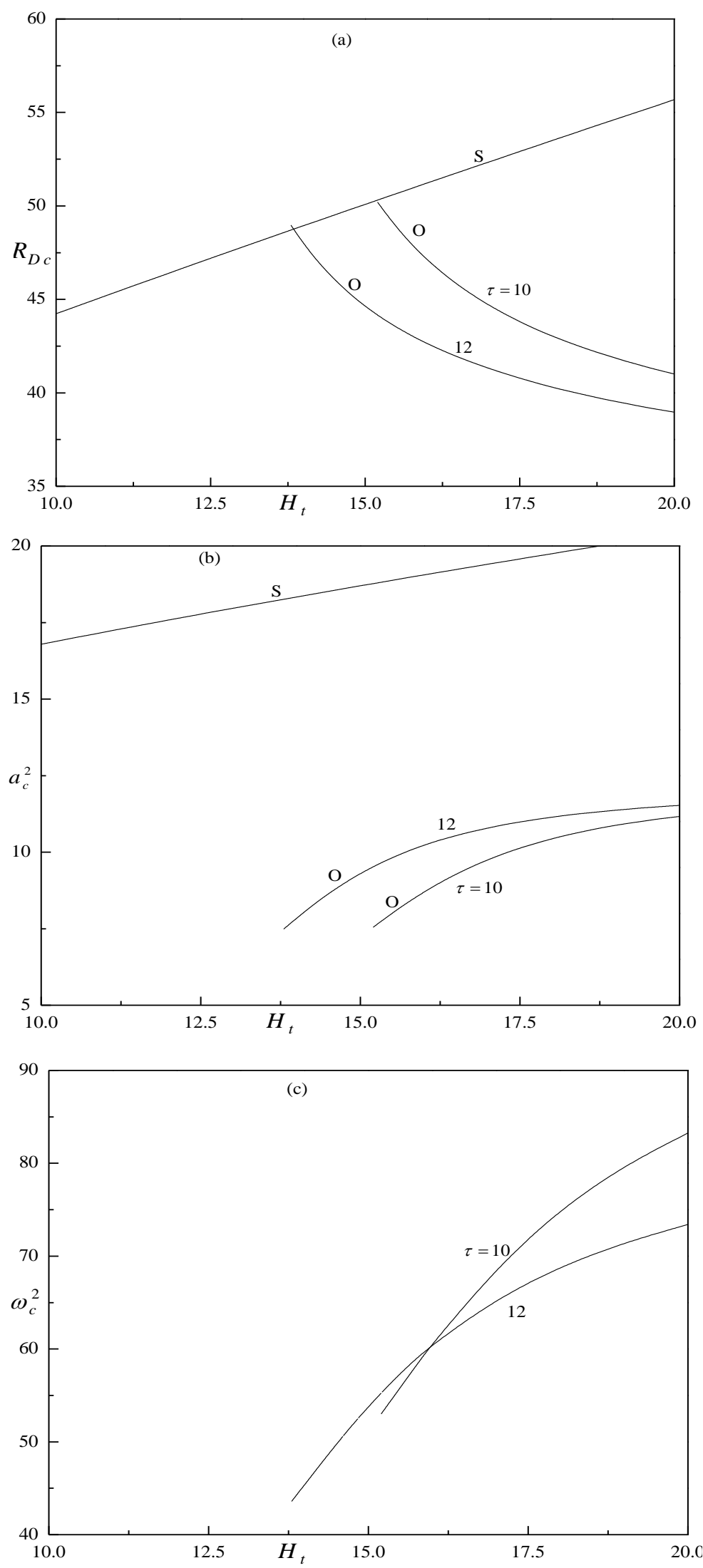

Fig. 7 Variation of (a) $R_{D c}$, (b) $a_{c}^{2}$ (c) $\omega_{c}^{2}$ with $H_{t}$, porous skeleton made of $\mathrm{Al}_{2} \mathrm{O}_{3}$ for two values of $\tau$ with $M_{1}=0.5$ and $M_{3}=1$. 


\begin{tabular}{|c|c|c|c|c|c|c|}
\hline$M_{1}$ & $\tau$ & $R_{D c}^{s}$ & $R_{D c}^{O}$ & $a_{c}^{2}$ & $\omega_{c}^{2}$ & $H_{t}^{T}$ \\
\hline \multirow{12}{*}{0} & \multirow{4}{*}{0.5} & 51.4717 & & 11.7549 & & 9 \\
\hline & & 52.3596 & & 11.8085 & & 10 \\
\hline & & & 51.6612 & 11.1435 & 3.04151 & 11 \\
\hline & & & 51.0431 & 11.2594 & 5.25999 & 12 \\
\hline & \multirow{4}{*}{1} & 47.2367 & & 11.3397 & & 5 \\
\hline & & 48.4147 & & 11.4821 & & 6 \\
\hline & & & 47.0621 & 10.4643 & 3.25119 & 7 \\
\hline & & & 46.3464 & 10.5938 & 4.69445 & 8 \\
\hline & \multirow{4}{*}{10} & 39.4784 & & 9.8696 & & 0 \\
\hline & & 41.3621 & & 10.3126 & & 1 \\
\hline & & & 41.1084 & 9.8912 & 0.66283 & 2 \\
\hline & & & 40.5969 & 9.9529 & 0.842401 & 3 \\
\hline \multirow{12}{*}{0.5} & \multirow{4}{*}{0.5} & 40.0787 & & 14.3567 & & 9 \\
\hline & & 40.7502 & & 14.4384 & & 10 \\
\hline & & & 40.4659 & 13.3834 & 2.8879 & 11 \\
\hline & & & 39.9368 & 13.5598 & 5.64275 & 12 \\
\hline & \multirow{4}{*}{1} & 36.9165 & & 13.7875 & & 5 \\
\hline & & 37.7897 & & 13.9754 & & 6 \\
\hline & & & 37.0868 & 12.5915 & 3.20576 & 7 \\
\hline & & & 36.4721 & 12.7989 & 5.03109 & 8 \\
\hline & \multirow{4}{*}{10} & 31.276 & & 11.9940 & & 0 \\
\hline & & 32.6286 & & 12.5173 & & 1 \\
\hline & & & 32.5644 & 11.9871 & 0.694873 & 2 \\
\hline & & & 32.1363 & 12.0875 & 0.924355 & 3 \\
\hline \multirow{12}{*}{1} & \multirow{4}{*}{0.5} & 32.5135 & & 16.0927 & & 9 \\
\hline & & 33.0471 & & 16.1923 & & 10 \\
\hline & & & 32.9698 & 14.8707 & 2.63031 & 11 \\
\hline & & & 32.5119 & 15.0959 & 5.80113 & 12 \\
\hline & \multirow{4}{*}{1} & 30.0191 & & 15.4341 & & 5 \\
\hline & & 30.7049 & & 15.6473 & & 6 \\
\hline & & & 30.3244 & 14.0264 & 3.08234 & 7 \\
\hline & & & 29.7899 & 14.3009 & 5.21651 & 8 \\
\hline & \multirow{4}{*}{10} & 26.6831 & & 14.0412 & & 1 \\
\hline & & 27.6259 & & 14.4956 & & 2 \\
\hline & & & 26.3354 & 13.5781 & 0.98024 & 3 \\
\hline & & & 26.2008 & 13.5944 & 1.05184 & 4 \\
\hline
\end{tabular}

Table 1 Critical Darcy Rayleigh number, critical wave number for different values of solid thermal relaxation time parameter $\tau$ and magnetic number $M_{1}$ when $M_{3}=1, \alpha=1$ and $\gamma=1$. 